\title{
Geiger-mode avalanche photodiodes as photodetectors in Cherenkov astronomy
}

Thomas KraehenbuehI*1, Hans Anderhub ${ }^{1}$, Michael Backes ${ }^{2}$, Adrian Biland ${ }^{1}$, Andrea Boller ${ }^{1}$, Isabel Braun ${ }^{1}$, Thomas Bretz ${ }^{3}$, Sebastian Commichau ${ }^{1}$, Volker Commichau $^{1}$, Daniela Dorner ${ }^{1,4}$, Adamo Gendotti ${ }^{1}$, Oliver Grimm ${ }^{1}$, Hanspeter von Gunten ${ }^{1}$, Dorothée Hildebrand ${ }^{1}$, Urs Horisberger ${ }^{1}$, Daniel Kranich ${ }^{1}$, Eckart Lorenz $^{1,5}$, Werner Lustermann ${ }^{1}$, Karl Mannheim ${ }^{3}$, Dominik Neise ${ }^{2}$, Felicitas Pauss ${ }^{1}$, Dieter Renker ${ }^{6}$, Wolfgang Rhode ${ }^{2}$, Michael Rissi ${ }^{1}$, Sebastian Rollke ${ }^{2}$, Ulf Röser ${ }^{1}$, Luisa Sabrina Stark ${ }^{1}$, Jean-Pierre Stucki ${ }^{1}$, Gert Viertel ${ }^{1}$, Patrick Vogler ${ }^{1}$ and Quirin Weitzel $^{1}$

${ }^{1}$ Institute for Particle Physics, ETH Zurich, 8093 Zurich, Switzerland

${ }^{2}$ TU Dortmund University, 44227 Dortmund, Germany

${ }^{3}$ University of Würzburg, 97074 Würzburg, Germany

${ }^{4}$ ISDC Data Center for Astrophysics, University of Geneva, 1290 Versoix, Switzerland

${ }^{5}$ Max Planck Institute for Physics, 80805 Munich, Germany

${ }^{6}$ Paul Scherrer Institute, 5232 Villigen, Switzerland

E-mail: thomas.kraehenbuehlephys.ethz.ch

The progress in the development of Geiger-mode avalanche photodiodes (G-APD) has resulted in devices which show great promise for Imaging Atmospheric Cherenkov Telescopes (IACT). In the course of the First G-APD Camera Test (FACT) project with the purpose to construct a G-APD based camera, their characteristics are analysed and measured in detail. The angle dependence of the photon detection efficiency was measured and found to be flat.

The effects of saturation and cross-talk on the reconstruction of the number of detected photons was studied. Since these effects are of a statistical nature, the reconstructed number is limited in its precision. For small numbers of photons, crosstalk is the limiting factor. For photon numbers comparable to or higher than the number of cells of the G-APD, the main limitation is the saturation.

International Workshop on New Photon Detectors

June 24-26, 2009

Shinshu University Matsumoto Japan

\footnotetext{
* Speaker.
} 


\section{Introduction}

Photons of cosmic sources with energies above some $\mathrm{GeV}$ can either be measured directly by satellite experiments such as FERMI or indirectly by ground-based telescopes. When entering the atmosphere, these photons cause a shower of secondary particles which emit Cherenkov light. This light can be measured by so-called Imaging Air Cherenkov Telescopes (IACT). The camera of such a telescope makes high demands on the used photodetector, in particular concerning the necessary sensitivity and speed.

Today's IACTs such as MAGIC, H.E.S.S. and VERITAS use photomultiplier tubes for this task. Since Geiger-mode Avalanche Photodiodes (G-APD) [1] had a very rapid development in the past few years, their usability for IACTs is currently being investigated in the First G-APD Camera Test (FACT) project [2].

\section{Angle dependence}

To eliminate dead space in the camera between the sensitive areas of the G-APDs, light catchers (so-called Winston cones) are used. Two types of cones are discussed: hollow cones using a reflective foil and solid cones, which allow higher concentration ratios with the cost of high incidence angles at the chip surface [3]. An important requirement for the use of such cones is thus a high photon detection efficiency at large incidence angles.

The G-APD chip surface is usually covered by a protective layer, typically epoxy or silicon rubber. Any incident light beam is refracted at this layer towards smaller incidence angles $\alpha$ on the chip surface according to Snell's law. In order to measure at large incidence angles the protective cover was removed using sulfuric acid.

The diode was attached to a low-noise amplifier on a pivotable mounting (see figure 1, left side). A pulsed LED is used as a light source and to trigger the readout electronics. For short pulses, the number of detected photons is approximately Poisson distributed. The number of events where no photon is detected is $N_{p e d}$, the total number of events is $N_{t o t}$. The Poisson mean of the number of detected photons can then be calculated:

$$
\mu=-\ln \left(\frac{N_{p e d}}{N_{t o t}}\right)
$$

Saturation and crosstalk cause a deviation from the Poisson distribution. As these effects do not affect $N_{p e d}$, the mean value as calculated above remains correct.

The measured mean values are normalized to the value measured for a perpendicular light beam $(\alpha=0)$. The measured mean values decrease with $\cos (\alpha)$ for geometrical reasons, any angle dependence of the photon detection efficiency would cause a deviation. The chip is embedded in a ceramic package with a rim which causes shadowing for angles above $60^{\circ}$.

The measurement was realized with a very high precision of approximately one percent for two wavelengths $(450 \mathrm{~nm}$ and $564 \mathrm{~nm})$. No indication of an angle dependence of the photon detection efficiency was found (see figure 1 , right side). 

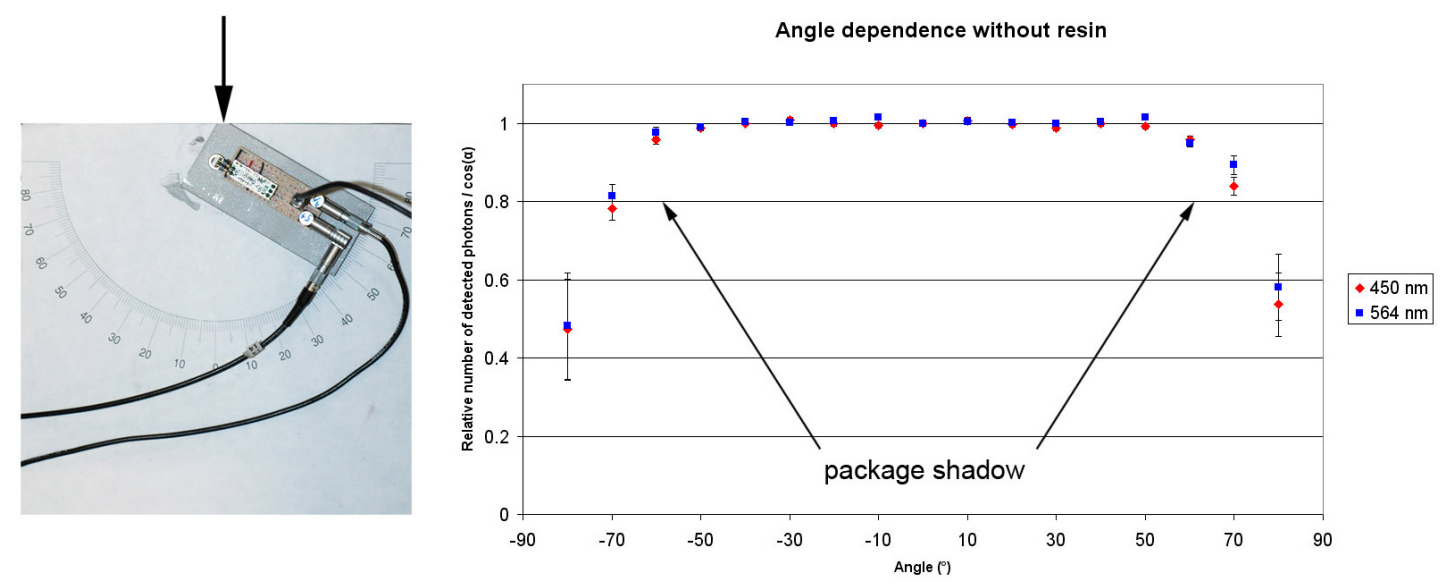

Figure 1: [Left] Setup used for the measurement of the angle dependence of the photon detection efficiency. A pulsed LED sends short light pulses in the direction indicated by the arrow, where the G-APD is mounted. [Right] Poisson mean of the number of detected photons divided by $\cos (\alpha)$. For small angles, the photon detection efficiency is independent of the incidence angle within $1 \%$.

\section{Reconstruction precision}

\subsection{Introduction}

When a light flash is detected by a G-APD, the resulting signal is influenced by several effects of statistical nature: saturation, crosstalk, afterpulses, dark counts and variations in the charge released per G-APD cell with a breakdown. Those effects induce noise to a measurement which exceed the unavoidable Poisson fluctuations of the number of incoming photons. For a certain constant size of light flashes, the number of photons impinging on the sensor per measurement is given by a Poisson distribution with mean $N_{i n c}$. Multiplying this value with the photon detection efficiency (PDE) gives the mean signal which would be measured by a noiseless sensor: $N=$ $P D E \times N_{\text {inc }}$. Since the signals are also Poisson distributed, the standard deviation is given by $\sqrt{N}$. A real measured signal $\tilde{N}$ includes additional fluctuation components. By correcting known nonlinearities a reconstructed value $N_{\text {rec }}$ is calculated. The standard deviation of the reconstructed values must be larger than $\sqrt{N_{\text {rec }}}$. Comparing the standard deviation including fluctuations with the minimal standard deviation of the Poisson distribution allows to quantify the effect of the individual noise components.

\subsection{Calculation}

The calculation of the value $N_{\text {rec }}$ from a signal $\tilde{N}$ is necessary for the investigation of the various noise components. For a photomultiplier tube, the two values are identical. A G-APD is more complex in the calculation due to saturation, crosstalk, afterpulses and the recovery of cells. The constriction to the conditions of a Cherenkov telescopes allows a major simplification: the photons arrive within a time window which is smaller than the recovery time of single cells. In combination with a readout based on the pulse height this allows to ignore cell recovery and thus afterpulses. 
For such a configuration the mean G-APD signal $\tilde{N}$ is given by

$$
\begin{aligned}
\tilde{N} & =N_{0}+\underbrace{S\left(\frac{N_{\text {cells }}-N_{0}}{N_{\text {cells }}} u_{c t} N_{0}, N_{\text {cells }}-N_{0}\right)}_{\text {crosstalk incl. saturation }} \\
\text { with } N_{0} & =S\left(N, N_{\text {cells }}\right) .
\end{aligned}
$$

$S\left(N, N_{\text {cells }}\right)$ is the function describing the statistical saturation effect. It is given by

$$
S\left(N, N_{\text {cells }}\right)=N_{\text {cells }}\left(1-\left(1-\frac{1}{N_{\text {cells }}}\right)^{N}\right) \quad[4]
$$

or simplified by $S\left(N, N_{\text {cells }}\right)=N_{\text {cells }}\left(1-e^{-\frac{N}{N_{\text {cells }}}}\right) . \quad u_{\text {ct }}$ is the mean number of cells triggered by crosstalk if only one cell initially has a breakdown (i.e. no saturation effects occur). The equations can be numerically solved for $N$ to get $N_{\text {rec }}$ as a function of $\tilde{N}$.

\subsection{Noise components of a G-APD}

The number of cells with a breakdown for a light flash has three fluctuation components: a Poissonian, one from the saturation and one due to crosstalk. Additionally, the charge released for a certain number of cells varies: the width of a histogram peak corresponding to $n$ triggered cells is given by $\Delta_{n}=\sqrt{\Delta_{p e d}^{2}+n \cdot \Delta_{a v}^{2}}$. $\Delta_{p e d}$ is the width of the pedestal peak with contributions from electronic noise and dark counts, $\Delta_{a v}$ is the contribution from fluctuations in the charge released per breakdown. Figure 2 (left) gives an overview of the described phenomena.

\subsection{Simulation specification}

A simple statistical Monte Carlo simulation was used to investigate the influence of the various noise components. The G-APD is described by an array of cells which are either active or in breakdown state. If a cell is switched from active to breakdown state, a neighbouring cell can be triggered by crosstalk with a probability $p_{c t}$ (recursively). A Poisson distributed number of times (mean $N$ ) a cell is selected one after another and triggered. The signal is then blurred according to the previous section by selecting a random value of a Gaussian distribution around the total number of triggered cells $n$ with width $\Delta_{n}$. Those values are the simulated signals $\tilde{N}$. For each $\tilde{N}$ the corresponding value $N_{\text {rec }}$ is calculated and their mean value and standard deviation $\Delta N_{\text {rec }}$ determined.

For a fixed number of cells $N_{\text {cells }}=900$ and several parameter sets $p_{c t}, \Delta_{p e d}$ and $\Delta_{a v}$ the simulation is run for a range of $N$. The performance of the simulation is checked by comparing simulated spectra with measured ones.

To compare the total standard deviation with the minimal (Poissonian) standard deviation, the following definition of the excess noise factor $\mathrm{F}$ is used:

$$
\begin{gathered}
\frac{\Delta N_{r e c}}{N_{r e c}}=\sqrt{\frac{F}{N_{r e c}}} \\
\Rightarrow F=\frac{\Delta N_{r e c}^{2}}{N_{r e c}}
\end{gathered}
$$



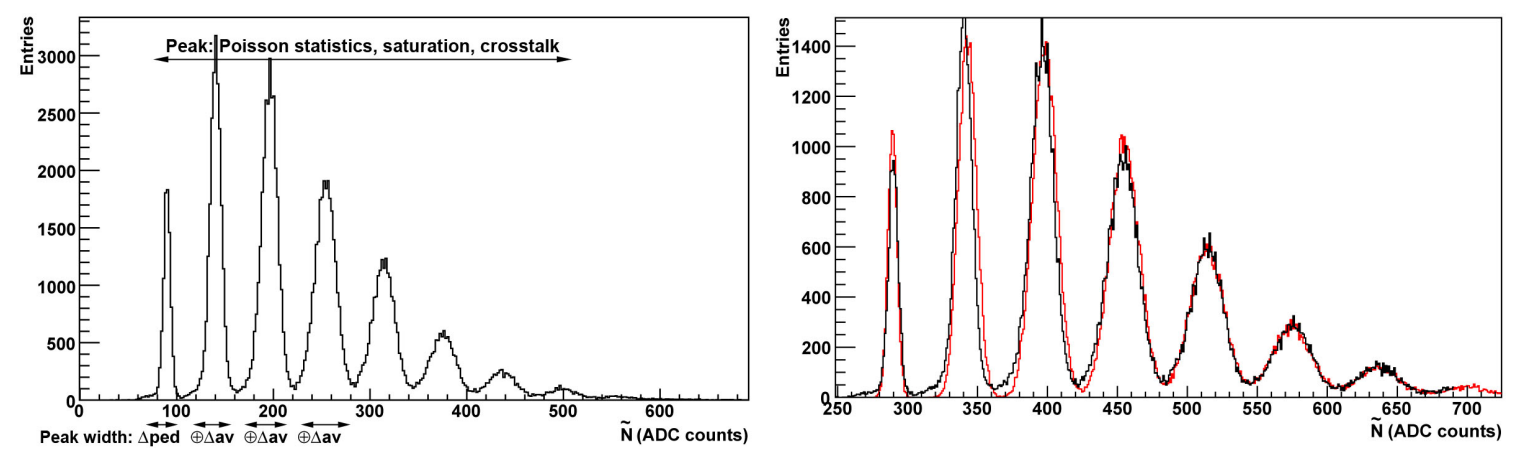

Figure 2: [Left] Overview of the fluctuation components of a G-APD. The number of incoming photons (Poisson distributed), together with the photon detection efficiency, saturation and crosstalk defines the number of cells with a breakdown and thus the peak. The peak width is caused by electronic noise and dark counts (pedestal width $\left.\Delta_{\text {ped }}\right)$ and variations in the charge released per breakdown $\left(\Delta_{a v}\right)$. [Right] Comparison of a measured spectrum (black) with the simulation (red). The two spectra agree with high accuracy.

Using this definition, the excess noise factor is one for a noiseless detector. For fluctuation components which are proportional to $\sqrt{N}$ (such as $\Delta_{a v}$ if crosstalk is neglected) the excess noise factor is independent of $N$ and is a measure for the noise characteristic of a detector. Both the pedestal noise $\Delta_{p e d}$ and the saturation fluctuations are not proportional to $\sqrt{N}$. The pedestal noise is independent of $N$ and the saturation fluctuations are disproportionally high for $N>N_{\text {cells }}$. The excess noise factor can be approximated by

$$
F \approx 1+p_{c t} \quad[1]
$$

when neglecting $\Delta_{a v}$ and saturation; when neglecting crosstalk and saturation the excess noise factor is

$$
F \approx 1+\Delta_{a v}^{2} . \quad[5]
$$

\subsection{Results}

The simple statistical simulation yields a very good agreement with measured spectra (see figure 2, right side). The fluctuation in the reconstructed number $N_{\text {rec }}$ is dominated by the Poisson statistics. Looking at the excess noise factor, the formulas 3.6 and 3.7 can be confirmed for small $N$, i.e. $N<<N_{\text {cells }}$ where saturation can be neglected (figure 3). As the fluctuations induced by saturation get disproportionally larger with increasing $N$, the excess noise factor according to the definition above is not a constant. For large $N$ (in the order of $N_{\text {cells }}$ or larger), the statistical noise eventually becomes larger than the Poisson variations.

\section{Outlook}

The current results of both the angle dependence measurement and the simulation encourage further research. It is planned to extend the measurement of the angle dependence to angles which are at the moment shadowed by the package. The analysis of the measurements will be extended to include surface reflections. The results from the simulation will be quantified and the combination of several components studied, especially concerning saturation. In parallel to the characterisation 


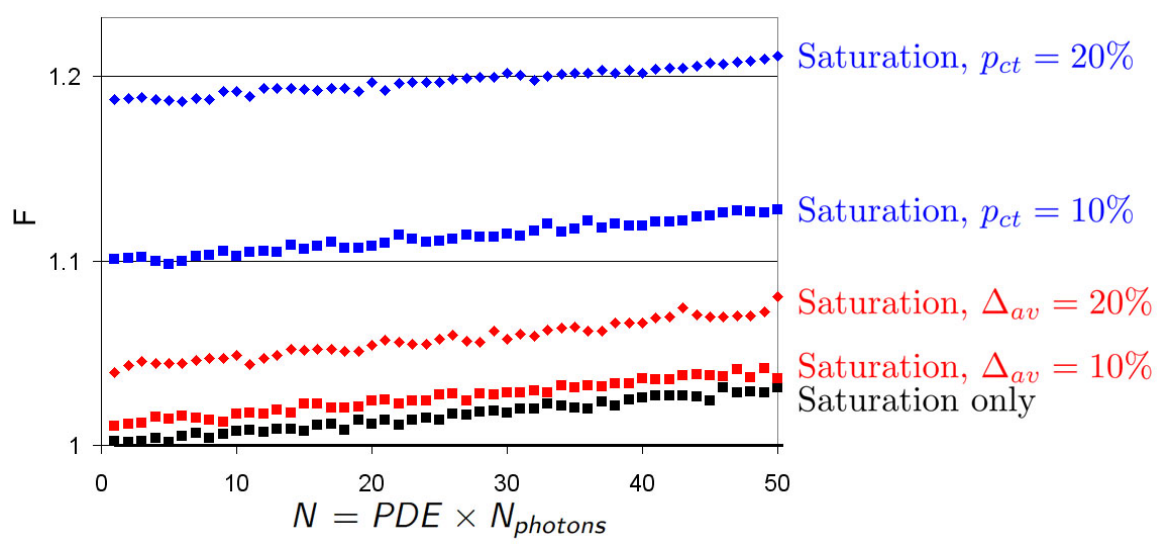

Figure 3: Excess noise factors for various simulation parameters: the excess noise factor depends on $N$ if only saturation effects are included (black). The same dependence on $N$ is seen when including $\Delta_{a v}$ (red, $10 \%$ and 20\%) or crosstalk (blue, $10 \%$ and 20\%). The limits for $N \rightarrow 0$ agree with the predictions. For typical values of $\Delta_{a v}=10 \%$ and a crosstalk probability of $10 \%$ the dominant contribution to the excess noise factor and thus to the precision of the reconstructed values $N_{\text {rec }}$ is the crosstalk for small $N$ and saturation for large $N$.

of G-APDs the FACT project is developing a full-sized camera based on the experience gained with the prototype module M0. The camera is foreseen for the DWARF telescope [6], a refurbished telescope of the HEGRA experiment located at La Palma (Spain). The telescope will be dedicated to the long-term monitoring of several bright sources in the very high energy range [7].

\section{References}

[1] D. Renker and E. Lorenz, Advances in solid state photon detectors, JINST 4 P04004, (2009).

[2] H. Anderhub et al., A novel camera type for very high energy gamma-ray astronomy based on Geiger-mode avalanche photodiodes, submitted to JINST, (2009).

[3] I. Braun et al., Solid Light Concentrators for Cherenkov Astronomy, in: Proceedings of the 31st ICRC (icrc1248), Łódź (2009)

[4] A. Stoykov et al., On the limited amplitude resolution of multipixel Geiger-mode APDs, JINST 2 P06005, (2007).

[5] Y. Musienko et al., The gain, photon detection efficiency and excess noise factor of multi-pixel Geiger-mode avalanche photodiodes, Nucl. Instr. Meth. A, 567, 57, (2006).

[6] T. Bretz et al., Status of the DWARF project for long-term monitoring of bright blazars, in: Proceedings of the 31st ICRC (icrc1257), Łódź (2009)

[7] M. Backes et al., Long-term monitoring of blazars - the DWARF network, in: Proceedings of the 31st ICRC (icrc1452), Łódź (2009) 